\section{A ATUAÇÃO DA EDUCAÇÃO FÍSICA NAS RESIDÊNCIAS MULTIPROFISSIONAIS EM SAÚDE}

\section{Performance of physical education in multiprofessional health residency}

\author{
La actuación de la educación física en las residencias \\ multiprofesionales en salud
}

\section{RESUMO}

Objetivo: Apresentar as atividades de competência e atuação da Educação Física nas residências multiprofissionais em saúde e as dificuldades dessa experiência. Síntese dos dados: Apresentam-se as atividades de competência e atuação no Sistema Único de Saúde (SUS) da Residência Integrada Multiprofissional Hospitalar com Ênfase na Atenção à Saúde Cardiometabólica do Adulto (RIMHAS) e da Residência Multiprofissional em Saúde da Família (RMSF), supervisionadas pela Comissão das Residências Multiprofissionais (COREMU), ambas com atenção à saúde dos usuários do SUS e duração de dois anos. No que diz respeito à atuação dos residentes, no primeiro ano na RIMHAS, as atividades são desenvolvidas no Hospital Universitário da Universidade Federal do Rio Grande (FURG), no Serviço de Pronto Atendimento, Clínica Médica e Clínica Cirúrgica; no segundo, ocorrem no Centro Integrado de Diabetes e Centro de Reabilitação Física. Na RMSF, há uma imersão em uma equipe de Estratégia de Saúde da Família, com atuação em uma Unidade Básica de Saúde, além da realização de estágios em outros serviços. Conclusão: O estudo mostra que experiências que articulam a formação acadêmica do núcleo da Educação Física e o SUS contribuem para a construção de um canal de diálogo entre indivíduos atuantes nas residências multiprofissionais.

Descritores: Educação Física e Treinamento; Promoção da Saúde; Pessoal Técnico de Saúde; Sistema Único de Saúde.

\section{ABSTRACT}

Objetive: To present the activities under the competence of physical education and its performance in the multidisciplinary residences in health and the difficulties involved in this experience. Data synthesis: The study presented the activities of competence and the performance in the Unified Health System (Serviço Unico de Saúde - SUS) of the Hospital Multiprofessional Integrated Residency Program with emphasis on Adults' Cardiometabolic Healthcare (Residencia Integrada Multiprofissional Hospitalar - RIHMAS) and the Multiprofessional Residency Program in Family Health (Residencia Multiprofissional em Saúde da Familia - RMSF), supervised by the Commission of Multidisciplinary Residences (Comissão das Residencias Multiprofissionais - COREMU), both with two-year duration, aiming healthcare for SUS users. As regards the actions of residents, all activities take place at the University Hospital of the Federal University of Rio Grande (FURG); in their first year in RIMHAS, the activities are developed in the Emergency room, Medical and Surgical Clinic; in the second one, they occur in the Integrated Center for Diabetes and Physical Rehabilitation Centre. In RMSF there is a dip in the Family Health Strategy, carrying out activities in a Basic Health Unit, in addition to internships in other health services. Conclusion: The study shows that experiences that link the academic training of the Physical Education core and the SUS contribute to open a channel of dialogue between individuals acting in multiprofessional residency programs.

Descriptors: Physical Education and Training; Health Promotion; Allied Health Personnel; Unified Health System.
Descrição ou avaliação de experiências, métodos, técnicas, procedimentos e instrumentais

\author{
Leandro Quadro Corrêa $a^{(1,2)}$ \\ Mirella Pinto Valério ${ }^{(1,3)}$ \\ André Oliveira Teixeira ${ }^{(2)}$ \\ Luis Fernando Guerreiro ${ }^{(2)}$ \\ Daniel Fossati Silveira ${ }^{(2)}$ \\ Pitágoras Terra Machado ${ }^{(2)}$ \\ Bruno Ezequiel Botelho Xavier ${ }^{(2)}$ \\ Manoela Maciel Oliz ${ }^{(2)}$ \\ Diogo Silveira Heredia Y \\ Antunes $^{(3)}$ \\ Alan Goularte Knuth ${ }^{(1,3)}$
}

1) Universidade Federal do Rio Grande Rio Grande do Sul (RS) - Brasil.

2) Residência Integrada Multiprofissional Hospitalar com Ênfase na Atenção à Saúde Cardiometabólica do Adulto (RIMHAS) Hospital Universitário Miguel Riet Corrêa Jr. - Universidade Federal do Rio Grande - Rio Grande do Sul (RS) - Brasil.

3) Residência Multiprofissional em Saúde da Família (RMSF) - Hospital Universitário Miguel Riet Corrêa Jr. - Universidade Federal do Rio Grande - Rio Grande do Sul (RS) - Brasil.
Recebido em: 20/03/2014 Revisado em: 11/05/2014 Aceito em: 11/06/2014 


\section{RESUMEN}

Objetivo: Presentar las actividades de competencia y actuación de la Educación Física en las residencias multiprofesionales en salud y las dificultades de esa experiencia. Síntesis de los datos: Se presentan las actividades de competencia y actuación en el Sistema de Salud (SUS) de la Residencia Integrada Multiprofesional Hospitalario con Énfasis en la Atención a la Salud Cardiometabólica del Adulto (RIMHAS) y de la Residencia Multiprofesional en Salud de la Familia (RMSF), supervisionadas por la Comisión de las Residencias Multiprofesionales (COREMU), ambas con atención a la salud de los usuarios del SUS y duración de dos años. Respecto la actuación de los residentes, en el primer año de la RIMHAS, las actividades son desarrolladas en el Hospital Universitario de la Universidad Federal de Rio Grande (FURG), en el Servicio de Pronta Asistencia, Clínica Médica y Clínica Quirúrgica. En el segundo año las actividades se dan en el Centro Integrado de Diabetes y Centro de Rehabilitación Física. En la RMSF hay una inmersión en un equipo de Estrategia de Salud de la Familia con actuación en una Unidad Básica de Salud, además de la realización de prácticas en otros servicios. Conclusión: El estudio muestra que las experiencias que involucran la formación académica del núcleo de Educación Física y el SUS contribuyen para la construcción del dialogo entre los individuos de las residencias multiprofesionales.

Descriptores: Educación y Entrenamiento Físico; Promoción de la Salud; Técnicos Medios en Salud; Sistema Único de Salud.

\section{INTRODUÇÃO}

As residências multiprofissionais em saúde são orientadas pelos princípios e diretrizes do Sistema Único de Saúde (SUS), a partir das necessidades e realidades locais e regionais. Elas abrangem as profissões da área da saúde: Biomedicina, Educação Física, Enfermagem, Farmácia, Fisioterapia, Fonoaudiologia, Nutrição, Odontologia, Psicologia, Serviço Social, Terapia Ocupacional, entre outras $^{(1)}$.

As residências multiprofissionais se apresentam num cenário de mudança de cuidados em saúde, em que aspectos como a promoção da saúde, a descentralização do cuidado médico e o trabalho multiprofissional são agregados em torno do saber fazer no campo da saúde, na perspectiva de contribuir para a integralidade do cuidado dos sujeitos. As residências multiprofissionais operam num cenário distinto do mencionado pelos que falam a favor ou contra sua proposta $^{(2)}$, sendo uma nova realidade para o SUS.

No que diz respeito à inserção dos trabalhadores de educação física nos programas de residências multiprofissionais em área da saúde, parece ser uma oportunidade única para que esses profissionais possam exercer mais uma função social relevante, além de ser uma alternativa que pode contribuir para a diminuição da distância entre o mundo acadêmico e a atuação profissional ${ }^{(3)}$.

Devido ao recente surgimento das residências com inclusão dos trabalhadores de educação física, é essencial divulgar essa experiência, a fim de contribuir com outros centros e novos cursos, para que possam ter um referencial que auxilie e contribua com sua constituição, tendo em vista também a escassez de literatura que trata especificamente do tema apresentado.

O presente estudo teve como objetivo apresentar as atividades de competência e atuação da Educação Física nas residências multiprofissionais e as dificuldades dessa experiência.

\section{SÍNTESE DOS DADOS}

Na Universidade Federal do Rio Grande (FURG), existem duas residências multiprofissionais em saúde: a Residência Integrada Multiprofissional Hospitalar com Ênfase na Atenção à Saúde Cardiometabólica do Adulto (RIMHAS) e a Residência Multiprofissional em Saúde da Família (RMSF), ambas com atenção à saúde dos usuários do SUS.

Os programas das residências multiprofissionais da FURG são supervisionados pela Comissão das Residências Multiprofissionais (COREMU), órgão que também representa a universidade junto à Comissão Nacional de Residência Multiprofissional em Saúde. A gestão das residências é partilhada com a Secretaria Municipal de Saúde. Essas residências objetivam a realização de atividades em nível de pós-graduação lato sensu, caracterizada por formação em serviço, e envolvem profissionais da área de Educação Física, Enfermagem e Psicologia.

\section{Das atividades específicas dos residentes de Educação Física na RIMHAS}

Os residentes do primeiro ano são denominados $\mathrm{R} 1 \mathrm{e}$ os do segundo ano, R2. Eles desenvolvem suas atividades práticas em diferentes campos no Hospital Universitário da FURG (HU/FURG), sob a supervisão de preceptores da área de Educação Física com titulação de mestre, contratados para supervisionar diretamente as atividades práticas dos residentes e responsáveis por promover a inserção destes nas equipes de saúde e a sua integração com outros serviços e com a população ${ }^{(4)}$. São também responsáveis pelo desenvolvimento do serviço em atenção aos pacientes cardiometabólicos e pelas atividades nos campos de atuação dos residentes em períodos nos quais estão envolvidos em atividades teóricas.

Noprimeiro ano de residência, os R1 têm sua inserçãonos ambientes de trabalho da clínica geral, onde desempenham 
suas funções no Serviço de Pronto Atendimento (SPA), Clínica Médica, Clínica Cirúrgica e pós-alta hospitalar. A justificativa de atuação nessas unidades é, primeiramente, implementar a residência multiprofissional nas áreas estratégicas para o SUS, estimulando o trabalho em equipe multiprofissional e contribuindo para a qualificação de recursos humanos especializados, a fim de garantir assistência integral à saúde - objetivo que vai ao encontro da proposta do programa Nacional de Reestruturação dos Hospitais Universitários Federais (ReHUF) ${ }^{(5)}$.

Nos ambientes hospitalares, a rotina dos residentes de Educação Física proporciona a aquisição de experiências frente ao funcionamento de um hospital universitário, onde acompanham a linha de cuidado do paciente e os ambientes onde ele receberá os devidos tratamentos. Paralelamente, espera-se que o residente desenvolva um olhar holístico frente aos problemas de saúde pública brasileira. Além disso, o trabalho multiprofissional permite que o paciente seja atendido na sua totalidade, e não apenas pelo motivo que o fez se internar, sendo realizada a assistência integral à saúde.

O primeiro ano de residência possui dois semestres, e os alunos são divididos em duas equipes, uma delas com campos de atuação nos setores de Clínica Cirúrgica e SPA e a outra, no atendimento da Clínica Médica. Estas equipes trocam de setores ao final do primeiro semestre. O SPA funciona 24 horas por dia, sendo na maioria das vezes o local de entrada do paciente no hospital (por urgência e/ ou descontrole de alguma patologia). A clínica cirúrgica é o setor que interna o paciente para as cirurgias, portanto, o atendimento ao paciente que está internado neste setor antecede a cirurgia ou é pós-cirúrgico.

Nos setores supracitados, o atendimento do residente é realizado quando há o encaminhamento do paciente pela equipe multiprofissional (médicos, fisioterapeutas, enfermeiros e psicólogos) ou pela avaliação dos pacientes internados. Quando não há encaminhamento, realizase o acolhimento, a assistência (preconizando a escuta do paciente, para que seja tratado com pessoalidade e individualidade) e a educação em saúde para o paciente e seus familiares. Basicamente, procede-se com uma conversa no leito sobre hábitos de vida e conversas com a equipe multiprofissional, para que a melhoria da vida do paciente seja um objetivo em comum.

Os residentes também realizam avaliação física e atividades de reabilitação física para pacientes que necessitem. Dependendo do caso, ainda pode ter continuidade o tratamento começado na internação, através do programa de atendimento pós-alta hospitalar. $\mathrm{Na}$ reabilitação física, após anamnese e discussão com o médico sobre medicamentos, realiza-se prescrição de exercício físico baseado no tempo de internação.
No segundo ano de residência, o serviço dos R2 ocorre em dois ambientes: Centro Integrado ao Diabetes (CID) e Centro de Reabilitação Física e Cardiometabólica HU-FURG (CRFC). São realizadas consultas em nível ambulatorial que contemplam avaliação física e educação em saúde, além de conversa terapêutica, com o intuito de conhecer o usuário, sua história, seus gostos, e prestarlhe atenção e acolhimento dignos. A avaliação física compreende: antropometria, hemodinâmica (pressão arterial, frequência cardíaca, saturação de oxigênio), força, flexibilidade, equilíbrio, avaliação cardiorrespiratória, além de questionários para determinar o risco cardiovascular e o índice de atividade física. Esse procedimento objetiva guiar a conduta, que é discutida de forma multiprofissional, e averiguar se há necessidade de exames complementares, como eletrocardiograma (ECG) e ECG de esforço, para inclusão ou não na reabilitação física.

O programa de reabilitação física ocorre no CRFC, tendo como foco principal os pacientes cardiometabólicos de alto risco. O trabalho desenvolvido pelos residentes está ancorado na Política Nacional de Humanização $(\mathrm{PNH})^{(6)}$ e possui caráter técnico-científico, uma vez que as atividades realizadas são baseadas em evidências ${ }^{(7-11)}$. Importante salientar que a visão de reabilitação multiprofissional nem sempre estará vinculada à cura, e sim em não fragmentar o indivíduo, buscando sua independência física, emocional e social, maximizando seu potencial funcional e melhorando sua qualidade de vida ${ }^{(12)}$. Busca-se um desfecho satisfatório, considerando não somente uma reabilitação biológica, mas também a integração satisfatória do indivíduo cardiometabólico na sua rotina familiar, social e profissional.

O programa de exercícios desenvolvidos no CRFC tem duração, normalmente, de um a seis meses, de acordo com a gravidade da doença e a evolução do paciente. A frequência semanal das sessões de reabilitação varia entre duas e três vezes. No período de maio de 2012 até julho de 2013, foram realizados 5.200 atendimentos pelos preceptores juntamente aos residentes de Educação Física da RIMHAS.

\section{Das atividades específicas do residente de Educação Física na RMSF}

Em função da organização proposta pelo Projeto Político-Pedagógico da RMSF da FURG ${ }^{(13)}$, a vivência dos residentes no primeiro ano se dá por meio de "imersão" em uma equipe de Estratégia de Saúde da Família (ESF), implicando em um contato intenso com esta, a população e o território dessa unidade. Durante o segundo ano, o residente mantém sua atuação na Unidade Básica de Saúde da Família (UBSF), mas passa a realizar também os estágios de vivência em outros serviços, tais como: Centro de Atendimento Psicossocial (CAPS), Núcleos de Apoio à Saúde da Família (NASF), Gestão, Vigilância Sanitária, 
entre outras possibilidades. O propósito dessa fase é aprofundar seu conhecimento sobre a rede de saúde e as possibilidades de interlocução da Educação Física nesses cenários.

O processo de trabalho na atenção básica é ainda desconhecido para a Educação Física, e as equipes de saúde ainda desconhecem os potenciais de atuação do núcleo da Educação Física na atenção, promoção, prevenção ou reabilitação da saúde ${ }^{(14-16)}$. Não há, até o momento, uma demanda específica para a Educação Física no campo da saúde, dessa forma, percebe-se uma nuvem de incertezas e desconhecimentos. Quando há demanda, ela se restringe ou se isola nas questões que envolvem o emagrecimento ou o “combate" à hipertensão e diabetes ${ }^{(17)}$.

Para esclarecer as reais possibilidades de atuação da Educação Física, surge a necessidade de um diálogo constante com a equipe da UBSF, buscando a criação de "fluxos" ou caminhos para que o usuário e/ou residente se encontre em um espaço de cuidado. Tais fluxos têm sido "desenhados" principalmente a partir de discussões de casos em conjunto com a equipe, que indicará se os sujeitos serão convidados a participar de um acolhimento ou de um grupo específico atendido pelos residentes de Educação Física da RMSF. Há também o incentivo constante de que a equipe convide os usuários a participar de espaços de promoção de saúde, de funcionamento variável e intrínseco à atuação dos residentes, como o grupo de tai chi chuan ou de emagrecimento. Além disso, uma pequena demanda espontânea chega à UBSF e necessita de um acolhimento diferenciado do que já é oferecido pela equipe, realizado pelo residente da Educação Física, muitas vezes em conjunto com seus colegas psicólogos ou enfermeiros.

Durante os acolhimentos, são pensadas, juntamente com o usuário, estratégias de cuidado que possam olhar seu problema de saúde de forma ampliada. Alguns desses acolhimentos resultaram no acompanhamento individual dos usuários - não no sentido de um treinamento físico direcionado, algo bastante presente na Educação Física, mas com sentido garimpado na prática dos serviços públicos de saúde. Tal espaço foi potente para a criação de vínculo, de escuta acolhedora, de olhar integral sobre o sujeito e de intervenção a partir das práticas corporais. Esse somatório de intenções procurou dar conta do problema de saúde trazido pelo sujeito, tanto em uma abordagem curativa, quando necessária, quanto na criação de novas possibilidades de vivência mais harmônicas e saudáveis. As abordagens individuais se davam na unidade de saúde e na residência dos sujeitos. Em geral, os casos acompanhados individualmente eram de sujeitos que, por alguma razão, não tinham indicação para participar de um grupo, devido à impossibilidade de se deslocar à unidade, a demandas bastante específicas ou a dificuldades de relacionamento, que exigiam, em um primeiro momento, uma abordagem individual.

Além dos acompanhamentos individuais, o residente em Educação Física promoveu um grupo de tai chi chuan, que se constituiu em um espaço de promoção de saúde aberto a toda a população queixosa, doente ou não. Promoveu, em conjunto com seus colegas, um grupo de apoio, espaço de partilha dos sofrimentos cotidianos dos indivíduos, buscando a criação de redes de apoio e vínculos fraternos, realizando dinâmicas que envolviam práticas corporais. Além dessas iniciativas, o residente participou também da manutenção da horta de plantas medicinais, condimentares e legumes, que fica ao lado da UBSF e foi criada pela primeira turma de residentes. É um espaço de relacionamento e de compartilhar mudas e saberes sobre as plantas medicinais e condimentares.

Outras possibilidades de atuação na vivência do residente em Educação Física, já institucionalizadas, seja pelo Estado brasileiro, seja por iniciativas da gestão local, são o trabalho em conjunto com a equipe do NASF, envolvendo-se com a lógica do matriciamento, ou, ainda, a participação no Projeto Vida Ativa, no qual são oferecidas, nas UBSFs do município, atividades de caminhada e ginástica para indivíduos hipertensos e diabéticos. Os residentes participam de atividades de controle social, acompanhando as reuniões do Conselho Municipal de Saúde. No cenário local da UBSF, participaram da criação e fortalecimento do Conselho Local de Saúde da UBSF São Miguel I. Também foi organizado um pequeno diagnóstico comunitário, a fim de se obter percepções da comunidade sobre a UBSF, referentes ao acesso e à qualidade da atenção. $\mathrm{O}$ diagnóstico não teve rigidez quanto à objetividade dos instrumentos, mas os resultados foram apresentados em reunião de equipe da UBSF. Também se tornou evidente a escassez de espaços sociais de convivência, lazer e organização da comunidade, assim como a pouca difusão do ideário de "controle social" enquanto ferramenta de construção do SUS, dado que ainda é nítido o afastamento da comunidade e a tomada de decisão nos serviços de saúde.

As experiências relacionadas à gestão no primeiro ano de residência se deram de forma mais intensa nos processos micropolíticos da própria UBSF, espaço de intensos conflitos, cujas intervenções buscavam contribuir para a organização do processo de trabalho da equipe e do fortalecimento de vínculos, respeito mútuo, fraternidade e confiança. Durante o segundo ano, abre-se a possibilidade de uma visão macro no cenário municipal, a partir da vivência na coordenação da saúde da família.

Dependendo da abertura dos profissionais que compõem os cenários políticos, há uma maior ou menor possibilidade de intervenção. Conseguiu-se, junto à SMS, contribuir para a criação da Política Municipal de Práticas Integrativas e 
Complementares, que tem como uma das ações a serem implementadas, as práticas corporais integrativas (yoga, tai chi chuan, lian gong, terapias corporais, biodança etc.).

Ainda que não seja uma atribuição isolada ou específica do residente, a própria gestão compartilhada da RMSF nesses anos iniciais de trajetória se configura como um espaço de gestão e troca de experiências entre professores da FURG, servidores da prefeitura e residentes. Para isso, a todo momento se fez necessário repensar a organização e o tempo imerso nas atividades, os conflitos entre as duas instâncias de gestão (universidade e prefeitura) e, dessa forma, esse espaço permeia a formação do residente, o qual encontra-se como resultante das capacidades e incapacidades das diferentes esferas na concepção de uma nova trajetória: a de formar residentes para o trabalho na saúde da família.

Outra importante instância de intervenção e formação do residente são as ações de educação permanente, as quais envolveram a participação em eventos como o II Extremos do Sul - Diálogos entre Educação Física e Saúde, as formações teóricas da RMSF, videoconferências etc. O residente tem a possibilidade de participar ativamente dos espaços e de propor temáticas a serem trabalhadas, como é o caso da Formação de Facilitadores em Lian Gong (Ginástica Chinesa), oferecida aos profissionais que atuam junto às UBSFs do município, com o intuito de ampliar essa prática na rede de saúde.

A intervenção/vivência, mesmo que seguindo o quadrilátero da formação ${ }^{(18)}$, varia muito dependendo do perfil de cada residente, de seus saberes e de com que ele se identifica. Varia também a partir da epistemologia identificada no curso de graduação, que origina a participação da Educação Física. Esse "não engessamento" da atuação pode apontar fragilidades, mas carrega um potencial rico de criação e liberdade para formações fortes, representativas e simbólicas, que expõe diferentes verdades, abrindo espaço para a experimentação e vivências singulares no cotidiano do SUS.

\section{Das dificuldades e avanços recentes}

Quando se coloca à frente do trabalho, evidenciam-se as dificuldades inerentes a esse processo. Sobram elementos causadores de imprecisão: a academia, a gestão política, as dificuldades de avançar em trabalho coletivo, a formação ainda fortemente disciplinar e calcada em regimes profissionais e de competências, as disputas particulares da Educação Física e a incapacidade do SUS de ser efetivado.

O modo como se prepara esta seção não tem uma disposição acadêmica, não está arraigado em uma avaliação formal, contudo, diz muito sobre uma dada realidade e um modo de saber-fazer particular ao núcleo de Educação Física no extremo sul do Brasil. Não se tem a intenção de criar esse mesmo sentido a outras realidades, mas compartilha-se esse saber a fim de encontrar certa ressonância. Residentes, em geral, recebem um contato para filiação ao conselho de Educação Física, e essa prática tem recebido certo respaldo do Ministério da Educação, apesar dos esforços em garantir a autonomia dos cursos de formação, em especial do curso de Educação Física da FURG, voltado à licenciatura. Tanto é realidade a ideia de que cabem debates sobre esses aspectos que a Portaria 256, de 11 de março de $2013^{(19)}$, estabelece ao NASF a compreensão de que o profissional de Educação Física abarca a alcunha de professor e bacharel, só para ficar à margem desse enorme debate que se expande. Em toda discussão, fica o compromisso de que o SUS não pode ser visto como um mercado para receber profissionais, e sim uma esfera pública com um sentido bastante singular de atuação.

A gestão da residência compartilhada entre universidade e prefeitura alimenta outra questão: os profissionais são suficientemente esclarecidos de qual papel o residente tem nos serviços de saúde? Tem-se observado que há um reducionismo ao fazer estritamente técnico, de modo que os residentes são recebidos para desafogar a elevada demanda de atividades, o que não é o caso. Dada a característica de formação dos residentes em serviço, é vital a defesa para que tenham a imersão que provoque a exata compreensão da realidade e o distanciamento que evidencia a capacidade de formulação para superação dos limites do serviço e a ampliação do conceito de saúde.

\section{CONCLUSÃO}

Através do exposto, é possível notar que existem diferenciações nas abordagens adotadas pela Educação Física no que tange às ações na RMSF e na RIMHAS, entretanto, esses espaços, dentro de suas especificidades, têm se tornado cenários possíveis para interlocução da Educação Física, seja na atenção primária ou secundária à saúde de usuários do SUS.

\section{REFERÊNCIAS}

1. Ministério da Saúde (BR), Conselho Nacionalde Saúde. Resolução nº 287, de 08 de outubro de 1998. Relacionar as categorias profissionais de saúde de nível superior para fins de atuação do Conselho. Diário Oficial da União [Internet]. 1998 [acesso em 2013 Ago 6]. Disponível em: http://portal.mec.gov.br/index. php?option $=$ com_content\&id $=12501 \&$ Itemid $=813$.

2. Dallegrave D, Kruse MHL. No olho do furacão, na ilha da fantasia: a invenção da residência multiprofissional em saúde. Interface Comun Saúde Educ. 2009;13(28): 213-37. 
3. Loch MR, Florindo AA. A Educação Física E As Residências Multiprofissionais Em Saúde. Rev Bras Ativ Fís Saúde. 2012;17(1):81-2.

4. Ministério da Educação (BR), Secretaria de Educação Superior, Comissão Nacional De Residência Médica Multiprofissional em Saúde. Resolução $\mathrm{n}^{0}$ 2, de 13 de abril de 2012. Dispõe sobre Diretrizes Gerais para os Programas de Residência Multiprofissional e em Profissional de Saúde. Diário Oficial da União, Brasília, 2012 Abr, n. 73 - Seção 1, p. 24-5

5. Presidência da República (BR), Casa Civil, Subchefia para Assuntos Jurídicos. Decreto $\mathrm{n}^{0} 7.082$, de 27 de janeiro de 2010. Institui o Programa Nacional de Reestruturação dos Hospitais Universitários FederaisREHUF, dispõe sobre o financiamento compartilhado dos hospitais universitários federais entre as áreas da educação e da saúde e disciplina o regime da pactuação global com esses hospitais. Diário Oficial da União, Brasília, 2010 Jan 27, Edição Extra.

6. Ministério da Saúde (BR), Secretaria-Executiva, Núcleo Técnico da Política Nacional de Humanização. HumanizaSUS - Política Nacional de Humanziação: a humanização como Eixo Norteador das Práticas de Atenção e Gestão em Todas as Instâncias do SUS. Brasília: Ministério da Saúde; 2004.

7. American College of Sports Medicine. Diretrizes do ACSM para os testes de esforço e sua prescrição. Rio de Janeiro: Guanabara Koogan; 2010.

8. Sociedade Brasileira de Cardiologia / Sociedade Brasileira DE Hipertensão / Sociedade Brasileira DE Nefrologia. VI Diretrizes brasileiras de hipertensão. Arq Bras Cardiol. 2010; 95(1 Supl 1):1-51.

9. Sociedade Brasileira de Diabetes. Diretrizes da Sociedade Brasileira de Diabetes 2013-2014 [acesso em 2014 Fev 2]. Disponível em: http://www.diabetes. org.br/diretrizes-idf

10. Sociedade Brasileira de Cardiologia. Diretriz de reabilitação cardiopulmonar e metabólica: aspectos práticos e responsabilidades. Arq Bras Cardiol. 2006;86(1):76-82.

11. Sociedade Brasileira de Cardiologia. Diretriz de Reabilitação Cardíaca. Arq Bras Cardiol. 2005;84(5):431-40.
12. Mendes EV. O modelo de atenção às condições crônicas na ESF. In: Mendes EV. O cuidado das condições crônicas na atenção primária à saúde: o imperativo da consolidação da estratégia da saúde da família. Brasília: Organização Pan-Americana da Saúde; 2012. p. 142-3.

13. Universidade Federal do Rio Grande. Projeto Político Pedagógico da Residência Multiprofissional em Saúde da Família. Rio Grande: Universidade Federal do Rio Grande; 2010.

14. Ministério da Saúde (BR), Secretaria de Atenção à Saúde. Portaria $n^{\circ}$ 154/2008 [Internet]. Brasília: Ministério da Saúde, 2013 [acesso em 2013 Ago 6]. Disponível em: http://www3.dataprev.gov.br/sislex/ paginas/66/MPS/2008/154.htm

15. Ministério da Saúde (BR), Secretaria de Vigilância em Saúde, Secretaria de Atenção à Saúde. Política nacional de promoção da saúde. Brasília: Ministério da Saúde; 2006.

16. Ministério da Saúde (BR), Secretaria de Atenção à Saúde. Portaria no 719/2011 [Internet]. Brasília: Ministério da Saúde, 2013 [acesso em 2013 Ago 6]. Disponível em: http://bvsms.saude.gov.br/bvs/ saudelegis/gm/2011/prt0719_07_04_2011.html

17. Bagrichevsky M, Palma A, Estevão A. Saúde Coletiva e Educação Física: aproximando campos, garimpando sentidos. In: Bagrichevsky M, Palma A, Estevão A, Rosa M. A saúde em debate na educação física. Blumenau: Nova Letra; 2006. p. 21- 43. v. 3.

18. Luz MT. Novos saberes e práticas em saúde coletiva: estudos sobre racionalidades médicas e atividades corporais. $3^{\mathrm{a}}$ ed. São Paulo: Hucitec; 2007.

19. Ministério da Saúde (BR), Secretaria de Atenção à Saúde. Portaria $n^{\circ}$ 256/2013 Brasília: Ministério da Saúde; 2013 [acesso em 2013 Ago 6]. Disponível em: http://bvsms.saude.gov.br/bvs/saudelegis/sas/2013/ prt0256_11_03_2013.html

\section{Endereço para correspondência:}

Leandro Quadro Corrêa

Universidade Federal do Rio Grande - FURG

Avenida Itália, $\mathrm{Km} 8$

Bairro: Carreiros

CEP: 96201-900 - Rio Grande - RS - Brasil

E-mail: leandroqc@hotmail.com 